\title{
GALOIS LINES FOR NORMAL ELLIPTIC SPACE CURVES, II
}

\author{
Hisao YoshiHaRa \\ Department of Mathematics, Faculty of Science, Niigata University, \\ Niigata 950-2181, Japan \\ E-mail:yosihara@math.sc.niigata-u.ac.jp
}

\begin{abstract}
For each linearly normal elliptic curve $C$ in $\mathbb{P}^{3}$, we determine Galois lines and their arrangement. The results are as follows: the curve $C$ has just six $V_{4}$-lines and in case $j(C)=1$, it has eight $Z_{4}$-lines in addition. The $V_{4}$-lines form the edges of a tetrahedron, in case $j(C)=1$, for each vertex of the tetrahedron, there exist just two $Z_{4}$-lines passing through it. We obtain as a corollary that each plane quartic curve of genus one does not have more than one Galois point.

key words and phrases : Galois line, space elliptic curve, Galois covering 2000 Mathematics Subject Classification number : 14H50, $14 \mathrm{H} 52$
\end{abstract}

\section{INTRODUCTION}

This is a continuation of [1], where we found three $V_{4}$-lines for each linearly normal elliptic curve $C$ in $\mathbb{P}^{3}$, and four $Z_{4}$-lines for such curve $C$ with $j(C)=1$. However, those lines are not all the ones. In this article we determine all Galois lines and describe their arrangement. First let us recall the definition of Galois lines briefly.

Let $k$ be the ground field of our discussion, we assume it to be algebraically closed, later we assume it the field $\mathbb{C}$ of complex numbers. Let $C$ be a smooth irreducible non-degenerate curve of degree $d$ in the projective three space $\mathbb{P}^{3}$ and $\ell$ a line in $\mathbb{P}^{3}$ not meeting $C$. Let $\pi_{\ell}: \mathbb{P}^{3} \rightarrow l_{0}$ be the projection with center $\ell$, where $\ell_{0}$ is a line not meeting $\ell$. Restricting $\pi_{\ell}$ to $C$, we get a surjective morphism $\left.\pi_{\ell}\right|_{C}: C \longrightarrow l_{0}$ and hence an extension of fields $\left(\left.\pi_{\ell}\right|_{C}\right)^{*}: k\left(\ell_{0}\right) \hookrightarrow k(C)$, where $\left[k(C): k\left(\ell_{0}\right)\right]=d$. Note that the extension of fields does not depend on $\ell_{0}$, but on $\ell$.

Definition 1. The line $\ell$ is said to be a Galois line for $C$ if the extension $k(C) / k\left(\ell_{0}\right)$ is Galois, or equivalently, if $\left.\pi_{\ell}\right|_{C}$ is a Galois covering. In this case $\operatorname{Gal}\left(k(C) / k\left(\ell_{0}\right)\right)$ is said to be the Galois group for $\ell$ and denoted by $G_{\ell}$.

If $\ell$ is the Galois line, then each element $\sigma \in G_{l}$ induces an automorphism of $C$ over $\ell_{0}$. We denote it by the same letter $\sigma$. Hereafter, assume $C$ is linearly normal, i.e., the hyperplanes cut out the complete linear series $\left|\mathcal{O}_{C}(1)\right|$. Then, the automorphism $\sigma$ can be extended to a projective transformation of $\mathbb{P}^{3}$, which will be also denoted by the same letter $\sigma$.

We use the following notation and convention:

- $V_{4}$ : the Klein 4-group

- $Z_{4}$ : the cyclic group of order four

$\cdot \sim$ : the linear equivalence of divisors

- $\operatorname{Aut}(C)$ : the automorphism group of $C$ 
- $\mathcal{L}(D):=\{f \in k(C) \backslash\{0\} \mid \operatorname{div}(f)+D \geq 0\} \cup\{0\}$, where $\operatorname{div}(f)$ is the divisor of $f$ and $D$ is a divisor on $C$.

$\cdot\langle\cdots\rangle$ : the group generated by the set $\{\cdots\}$ or the linear subvariety spanned by the set $\{\cdots\}$

- $V(F)$ : the variety defined by $F=0$

- $C \cdot H$ : the intersection divisor of $C$ and $H$ on $C$, where $H$ is a plane.

- $\ell_{P Q}$ : the line passing through $P$ and $Q$

\section{Statement of Results}

We assume $k=\mathbb{C}$ and use the same notation as in [1].

Definition 2. When $\ell$ is a Galois line for $C$ and $G_{\ell} \cong V_{4}$ (resp. $Z_{4}$ ), we call $\ell$ a $V_{4}$ $\left(\right.$ resp. $\left.Z_{4}\right)$-line.

There exist $V_{4}$-lines for the curve which is given by an intersection of hypersurfaces as follows.

Lemma 1. Suppose $S_{1}$ and $S_{2}$ are irreducible quadratic surfaces in $\mathbb{P}^{3}$ satisfying the following conditions:

(1) $S_{i}(i=1,2)$ has a singular point $Q_{i}$ and $Q_{1} \neq Q_{2}$.

(2) $S_{1} \cap S_{2}$ is a smooth curve $\Delta$.

(3) The line $\ell$ passing through $Q_{1}$ and $Q_{2}$ does not meet $\Delta$.

Then, $\Delta$ is a linearly normal elliptic curve and $\ell$ is a $V_{4}$-line for $\Delta$.

Let $C$ be a linearly normal elliptic curve in $\mathbb{P}^{3}$. Then, there exists a divisor $D$ of degree four on an elliptic curve $E$ such that $C$ is given by an embedding of $E$ associated with the complete linear system $|D|$. Note that $C$ can be expressed as an intersection of two quadratic surfaces.

Lemma 2. There exist just four irreducible quadratic surfaces $S_{i}(0 \leq i \leq 3)$ such that each $S_{i}$ has a singular point and contains $C$. Let $Q_{i}$ be the unique singular point of $S_{i}$. Then the four points are not coplanar.

Remark 3. Let $\pi_{Q}: \mathbb{P}^{3} \rightarrow \mathbb{P}^{2}$ be the projection with center $Q \in \mathbb{P}^{3} \backslash C$. If $\pi_{Q}$ induces a 2 to 1 morphism from $C$ onto its image in Lemma 2, then $Q$ coincides with one of $Q_{i}$.

The main theorem is stated as follows:

Theorem 1. For each linearly normal elliptic curve in $\mathbb{P}^{3}$, there exist four noncoplanar points $Q_{i}(0 \leq i \leq 3)$ such that the lines passing through each two of them are $V_{4}$-lines for $C$. Namely, all the $V_{4}$-lines form the six edges of a tetrahedron. Further, if the Weierstrass normal form of $E$ is given by $y^{2}=4\left(x-e_{1}\right)\left(x-e_{2}\right)\left(x-e_{3}\right)$, then we can present explicitly the coordinates of $Q_{i}$ (by taking a suitable coordinates of $\mathbb{P}^{3}$ ) as follows:

$$
Q_{0}=(0: 0: 0: 1) \text { and } Q_{i}=\left(1:-c_{i}: e_{i}: 0\right),(i=1,2,3),
$$

where $c_{i}=e_{i}^{2}+e_{j} e_{k}$ such that $\{i, j, k\}=\{1,2,3\}$. 


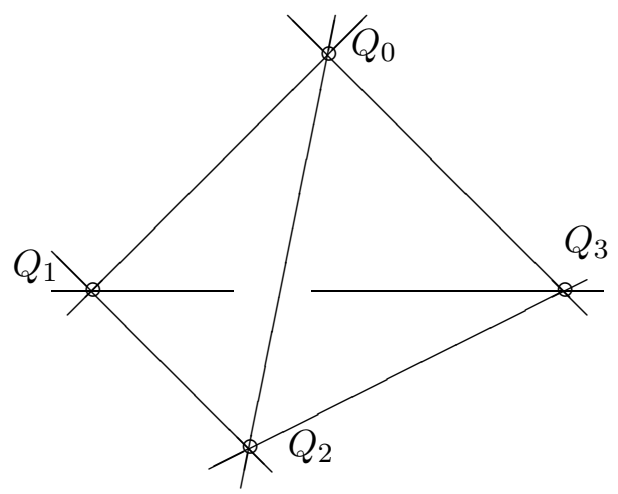

Remark 4. In the case of an elliptic curve $E$ in $\mathbb{P}^{2}$ it has a Galois point if and only if $j(E)=0$, and then it has just three $Z_{3}$-points.

In the case where the $j$-invariant $j(C)=1$, there exists an automorphism of order four with a fixed point. This curve has the other Galois lines as follows.

Theorem 2. Under the same assumption as in Theorem 1, if $j(C)=1$, then there exist eight $Z_{4}$-lines (in addition to the $V_{4}$-lines). To state in more detail, for each vertex $Q_{i}(0 \leq i \leq 3)$ of the tetrahedron in Theorem 1 , there exist two $Z_{4}$-lines passing through it. Therefore, for each vertex, there exist three $V_{4}$-lines and two $Z_{4}$-lines passing through it and the total number of Galois lines is fourteen. Two $Z_{4}$-lines do not meet except at one of the vertices.

Let $\Sigma$ be the set of six $V_{4}$-lines in Theorem 1. In the case where $j(C)=1$ let $\Sigma^{\prime}$ be the set of eight $Z_{4}$-lines in Theorem 2. The following corollary is an answer to the question for the case of outer Galois point [3, Theorem 2].

Corollary 5. For a plane quartic curve $\Gamma$ with genus one, the number of (outer) Galois points is at most one. If $\Gamma$ has the Galois point, then the Galois group $G$ is isomorphic to $V_{4}$ or $Z_{4}$. Further, if $G \cong V_{4}$ (resp. $\left.Z_{4}\right)$, then $\Gamma$ is obtained by a projection $\pi_{Q}: \mathbb{P}^{3} \rightarrow \mathbb{P}^{2}$ with center $Q$, where $Q \in \Sigma$ (resp. $\left.Q \in \Sigma^{\prime}\right)$ such that $Q \neq Q_{i}(0 \leq i \leq 3)$.

Remark 6. Different from the case of the space quartic curve, a plane quartic curve of genus one does not necessarily have a Galois point.

Remark 7 . Since $C$ is given by the embedding associated with a complete linear system and has a Galois line, the embedding is called a Galois embedding, which has been defined in [6].

\section{Proofs}

First we prove Lemma 1. It is easy to see that $\Delta$ has genus one and $\operatorname{dim} \mathrm{H}^{0}\left(\Delta, \mathcal{O}_{\Delta}(1)\right)=4$. Hence $\Delta$ is a linearly normal elliptic curve. Let $\pi_{Q_{i}}$ be the projection $\mathbb{P}^{3} \rightarrow P^{2}$ with center $Q_{i}(i=1,2)$ and put $\Delta_{i}=\pi_{Q_{i}}(\Delta) \subset \mathbb{P}^{2}$ and $R_{i}=\pi_{Q_{i}}\left(\ell \backslash\left\{Q_{i}\right\}\right)$. Then $\Delta_{i}$ is a conic and $R_{i}$ is a point not on $\Delta_{i}$. Let $\varpi_{R_{i}}$ be the projection $\mathbb{P}^{2} \rightarrow \mathbb{P}^{1}$ with center $R_{i}$. Restricting $\varpi_{R_{i}}$ to $\Delta_{i}$, we get a surjective morphism $\left.\varpi_{R_{i}}\right|_{\Delta_{i}}: C_{i} \longrightarrow \mathbb{P}^{1}$. Therefore we have two morphisms

$$
\pi_{i}=\varpi_{Q_{i}} \cdot \pi_{Q_{i}}: \Delta \longrightarrow \mathbb{P}^{1}
$$


of degree four. They coincide with the restriction of the projection $\pi_{\ell}: \mathbb{P}^{3} \rightarrow \rightarrow \mathbb{P}^{1}$. Note that $k\left(\Delta_{1}\right)$ and $k\left(\Delta_{2}\right)$ are distinct subfields of $k(\Delta)$ and $\left[k(\Delta): k\left(\Delta_{i}\right)\right]=$ $\left[k\left(\Delta_{i}\right): k\left(\mathbb{P}^{1}\right)\right]=2$. We infer that $k(\Delta)$ is a $V_{4}$-extension of $k\left(\mathbb{P}^{1}\right)$, hence $\left.\pi_{\ell}\right|_{\Delta}$ is a $V_{4}$-Galois covering. This proves Lemma 1.

Fix a universal covering $\pi: \mathbb{C} \longrightarrow \mathbb{C} / \mathcal{L}$, where $\mathcal{L}$ is the lattice in $\mathbb{C}$ defining a complex torus. We assume $\mathcal{L}=\mathbb{Z}+\mathbb{Z} \omega$, where $\Im \omega>0$. Let $\wp(z)$ be the Weierstrass $\wp$-function with respect to $\mathcal{L}$. Then, the map $\varphi: \mathbb{C} \longrightarrow E$ defined by

$\varphi(z)=\left(\wp(z): \wp^{\prime}(z): 1\right)$, induces an isomorphism $\bar{\varphi}: \mathbb{C} / \mathcal{L} \longrightarrow E$. The defining equation of the elliptic curve $E$ is the Weierstrass normal form $y^{2}=4 x^{3}+p x+q$. We assume it to be factored as $4\left(x-e_{1}\right)\left(x-e_{2}\right)\left(x-e_{3}\right)$. Put $P_{\alpha}=\varphi(\alpha)$ for $\alpha \in \mathbb{C}$. Denote by + the sum of divisors on $E$ and, at the same time, the sum of complex numbers. For example, $P_{\alpha}+P_{\beta}$ and $\alpha+\beta$ denote the sum of divisors and complex numbers respectively.

Lemma 8. We have the linear equivalence of divisors on E:

$$
P_{\alpha}+P_{\beta} \sim P_{\alpha+\beta}+P_{0} .
$$

Proof. This may be well-known. See, for example, [2, Ch. IV, Theorem 4,13B].

Lemma 9. Let $D$ be the divisor of degree four on $E$. By taking a suitable translation $\tau$ on $E$, we have $\tau^{*}(D) \sim 4 P_{0}$.

Proof. Suppose $D=\sum_{i=1}^{4} P_{\alpha_{i}}$. Then, take $\beta=-\sum_{i=1}^{4} \alpha_{i} / 4$. Let $\tau$ be the translation on $E$ induced from the one $z \mapsto z+\beta$ on $\mathbb{C}$. Then we have $\tau^{*}(D)=\sum_{i=1}^{4} P_{\alpha_{i}+\beta}$. Using Lemma 8 , we get $\tau^{*}(D) \sim 4 P_{0}$.

Let $D$ be a hypeplane section of $C$. Applying Lemma 9, we see that there exists an elliptic curve $C_{0}$ in $\mathbb{P}^{3}$ given by the embedding associated with $\left|4 P_{0}\right|$ and an isomorphism $\psi: \mathbb{P}^{3} \longrightarrow \mathbb{P}^{3}$ satisfying that $\psi\left(C_{0}\right)=C$ and $4 P_{0} \sim \psi^{*}(D)$. So that we have the following lemma.

Lemma 10. We can assume $C$ is given by the embedding associated with $\left|4 P_{0}\right|$.

Therefore it is sufficient for our purpose to consider the curve embedded by $\left|4 P_{0}\right|$. Let $\phi: E \longrightarrow C \subset \mathbb{P}^{3}$ be the embedding of $E$ associated with $\left|4 P_{0}\right|$.

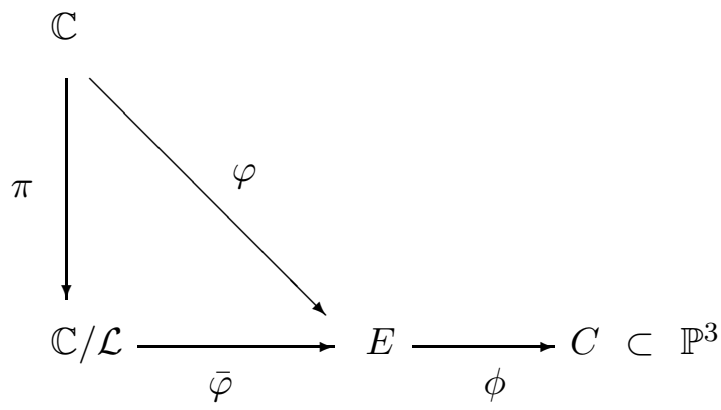

In order to study the number and arrangement of Galois lines, we provide some lemmas. Let $\mathcal{S}$ and $\mathcal{G}$ be the set of Galois lines for $C$ and the set of subgroups of 
$\operatorname{Aut}(C)$ respectively. Since a Galois line $\ell$ determine the Galois group $G_{\ell}$ in $\operatorname{Aut}(C)$ uniquely, we can define the following map.

Definition 3. We define an arrangement-map $\rho: \mathcal{S} \longrightarrow \mathcal{G}$ by $\rho(\ell)=G_{\ell}$.

We study the map $\rho$ in detail. Note that each element of $G_{\ell}$ can be extended to a projective transformation. That is, we have a faithful representation $r: G_{\ell} \longrightarrow$ $P G L(3, \mathbb{C})$.

Lemma 11. The map $\rho$ is injective.

Proof. For two elements $\ell_{i}$ of $\mathcal{S}(i=1,2)$, suppose $\rho\left(\ell_{1}\right)=\rho\left(\ell_{2}\right)$ and $\ell_{1} \neq \ell_{2}$. Then, the following two cases take place:

(i) $\ell_{1} \cap \ell_{2}$ consists of one point $P$.

(ii) $\ell_{1} \cap \ell_{2}=\emptyset$.

In the case (i), for a general point $Q \in C$, put $H_{i Q}=\left\langle\ell_{i}, Q\right\rangle(i=1,2)$ : the plane spanned by $\ell_{i}$ and $Q$. Since $G_{\ell_{1}}=G_{\ell_{2}}$, we have $H_{1 Q} \cap \ell_{0}=H_{2 Q} \cap \ell_{0}=\{R\}$, where $\ell_{0}$ is the line defined in Introduction. Further, since $\pi_{\ell_{1}}\left(H_{1 Q} \cap C\right)=\pi_{\ell_{2}}\left(H_{2 Q} \cap C\right)=R$, the set of four points $H_{1 Q} \cap C$ is equal to that of $H_{2 Q} \cap C$ and they lie on the line $H_{1 Q} \cap H_{2 Q}$, which passes through $P$. This implies $C$ is contained in the plane spanned by $\ell_{0}$ and $P$. Since $C$ is assumed to be non-degenerate, this is a contradiction. Next we treat the case (ii). Similarly, for a general point $Q \in C$, put $H_{i Q}=\left\langle\ell_{i}, Q\right\rangle$. Then, by the same argument as above, the four points $H_{1 Q} \cap C$ and $H_{2 Q} \cap C$ lie on the line $H_{1 Q} \cap H_{2 Q}$. Thus $C$ is contained in a rational normal scroll $\Sigma$. However, $H_{i Q} \cap \Sigma$ is a line, so that $\Sigma$ must be a plane. This is a contradiction.

We present a criterion when $G \subset \operatorname{Aut}(C)$ can be the image of an element of $\mathcal{S}$. See [6. Theorem 2.2] for a similar one. Hereafter we use the notation $P_{\alpha}^{\prime}=\phi\left(P_{\alpha}\right)=$ $(\phi \varphi)(\alpha) \in C$ for brevity.

Lemma 12. A subgroup $G=\left\{\sigma_{1}, \ldots, \sigma_{4}\right\}$ of $A u t(C)$ is an image of $\rho$ if and only if $G$ satisfies the following condition $(\diamond)$ :

$(\diamond)$ For each point $Q \in C$ the divisor $\sum_{i=1}^{4} \sigma_{i}(Q)$ is linearly equivalent to $4 P_{0}^{\prime}$ and $C / G$ is a rational curve.

Proof. If $G=\rho(\ell)$, then clearly $C / G \cong \mathbb{P}^{1}$. Take a plane $H$ satisfying that $H \supset \ell$ and $H \ni Q$. By definition the point $\sigma_{i}(Q)(1 \leq i \leq 4)$ lies on $H$, hence the divisor is linearly equivalent to $4 P_{0}{ }^{\prime}$. Conversely, for a point $Q \in C$, put $D=\sum_{i=1}^{4} \sigma_{i}(Q)$. By assumption we have $D \sim 4 P_{0}{ }^{\prime}$, hence $G$ acts on $\mathrm{H}^{0}\left(C, \mathcal{O}_{C}(1)\right)$. Therefore each element of $G$ can be extended to a projective transformation. Letting $\pi: C \longrightarrow$ $C / G \cong \mathbb{P}^{1}$, we take independent sections $s_{0}$ and $s_{1}$ of $\mathrm{H}^{0}\left(\mathbb{P}^{1}, \mathcal{O}_{\mathbb{P}^{1}}(1)\right)$ and put $\widetilde{s_{i}}=\pi^{*}\left(s_{i}\right)(i=1,2)$. Then we have $\sigma^{*}\left(\widetilde{s_{i}}\right)=\widetilde{s_{i}}$. Taking a basis of $\mathrm{H}^{0}\left(C, \mathcal{O}_{C}(1)\right)$ containing $\widetilde{s_{1}}$ and $\widetilde{s_{2}}$, we obtain a Galois line $\ell$ such that $\rho(\ell)=G$.

We study whether $\ell_{1} \cap \ell_{2}=\emptyset$ or $\neq \emptyset$ by observing $G_{\ell_{1}} \cap G_{\ell_{2}}$ in $\operatorname{Aut}(C)$.

Lemma 13. Suppose $\ell_{1}$ and $\ell_{2}$ are distinct Galois lines. Then, the following two cases take place.

(1) If $\ell_{1} \cap \ell_{2}=\emptyset$, then $G_{\ell_{1}} \cap G_{\ell_{2}}=\{\operatorname{id}\}$ in $\operatorname{Aut}(C)$.

(2) If $\ell_{1} \cap \ell_{2}$ is a point $P$, then it is a singular point of some quadratic surface containing $C$, Further, we have $G_{\ell_{1}} \cap G_{\ell_{2}}=\langle\sigma\rangle$, where $\sigma$ has order two and has a fixed point as an automorphism of $C$. 
Proof. Take an element $\sigma \in G_{\ell_{1}} \cap G_{\ell_{2}}$. It can be extended to a projective transformation. Since every plane $H_{i}$ containing $\ell_{i}$ is invariant by $\sigma$, we infer $\sigma\left(\ell_{i}\right)=\ell_{i}$ $(\mathrm{i}=1,2)$. Therefore, for each hyperplane $H_{1} \supset \ell_{1}$, if $H_{1} \cap \ell_{2}=\{Q\}$, then $\sigma(Q)=Q$, i.e., $\left.\sigma\right|_{\ell_{2}}=$ id. By the same argument we also have $\left.\sigma\right|_{\ell_{1}}=$ id. Since $\ell_{1} \cap \ell_{2}=\emptyset, \sigma$ is identity on $\mathbb{P}^{3}$. Next we treat the second case. Suppose $\ell_{1} \cap \ell_{2}$ consists of one point $P$. Then, for each point $Q \in C$, put $H_{i Q}=\left\langle\ell_{i}, Q\right\rangle$ and $\ell_{Q}=H_{1 Q} \cap H_{2 Q}$. Since $H_{i Q} \supset \ell_{Q}$ for $i=1$ and 2 , we have $\sigma(Q) \in \ell_{Q}$. Therefore $C$ is contained in the cone passing through $P$. Clearly the order of $\sigma$ is two. Since the quotient curve $C /\langle\sigma\rangle$ is isomorphic to $\ell_{0}$, the $\sigma$ has a fixed point in $C$.

From Lemma 13 we infer the following remark.

Remark 14. Let $\ell$ be a Galois line and take a point $P \in \ell$. Let $\pi_{P}: \mathbb{P}^{3} \rightarrow \mathbb{P}^{2}$ be a projection with center $P$. If $P$ is not the vertex of the tetrahedron, then $\pi_{P}(\ell \backslash\{P\})$ is a Galois point for the quartic curve $\pi_{P}(C)$. However, if $P$ is the one, then $\left.\pi_{P}\right|_{C}$ turns out to be a 2 to 1 morphism onto its image and $\pi_{P}(C)$ is a conic in $\mathbb{P}^{2}$.

Hereafter we denote by $\sigma_{i}(0 \leq i \leq 3)$ an automorphism of $E$ such that the representation on $\mathbb{C}$ is

$$
\sigma_{0}(z)=-z, \quad \sigma_{1}(z)=-z+\frac{1}{2}, \quad \sigma_{2}(z)=-z+\frac{\omega}{2}, \quad \sigma_{3}(z)=-z+\frac{1+\omega}{2} .
$$

Lemma 15. The number of $V_{4}$-lines is at most six.

Proof. Suppose $C$ has a $V_{4}$-line $\ell$. Then, let $H$ be a plane containing $\ell$ and $P_{0}{ }^{\prime}$. Since $\left.\pi_{\ell}\right|_{C}: C \longrightarrow \mathbb{P}^{1}$ is a $V_{4}$-covering, the intersection divisor $H \cdot C$ on $C$ can be expressed in one of the following two types:

(i) $H \cdot C=2 P_{0}{ }^{\prime}+2 P_{\gamma}^{\prime}$

(ii) $H \cdot C=P_{0}{ }^{\prime}+P_{\gamma_{1}}{ }^{\prime}+P_{\gamma_{2}}{ }^{\prime}+P_{\gamma_{3}}{ }^{\prime}$.

Suppose $G=\langle\sigma, \tau\rangle$, where

$$
\sigma(z)=-z+\alpha \text { and } \tau(z)=z+\beta
$$

on the universal covering $\mathbb{C}$, where $2 \beta \equiv 0(\bmod \mathcal{L})$ and $\beta \not \equiv 0(\bmod \mathcal{L})$. The case (i) $(\operatorname{resp} . \quad(i i))$ occurs when $\alpha \equiv 0(\bmod \mathcal{L})(\operatorname{resp} . \quad \alpha \not \equiv 0(\bmod \mathcal{L}))$ in $(1)$. We consider the possibility of $\alpha \neq \equiv$, i.e., we treat the case (ii). Since $H \cdot C$ is invariant by the action of $G$, it can be expressed as $P_{0}{ }^{\prime}+P_{\alpha}{ }^{\prime}+P_{\beta}{ }^{\prime}+P_{\alpha+\beta}{ }^{\prime}$. Since this is linearly equivalent to $4 P_{0}{ }^{\prime}$, we infer

$$
P_{\alpha}+P_{\beta}+P_{\alpha+\beta} \sim 3 P_{0}
$$

on $E$. The left hand side of (2) is linearly equivalent to $P_{2(\alpha+\beta)}+2 P_{0}$ by Lemma 8 , Therefore we have $P_{2(\alpha+\beta)} \sim P_{0}$. This implies $2(\alpha+\beta) \equiv 0(\bmod \mathcal{L})$, i.e., $2 \alpha \equiv 0$ $(\bmod \mathcal{L})$. Then, let us find the distinct subgroups $G$ of $\operatorname{Aut}(C)$ such that $G$ is generated by order two elements. By taking two from $\sigma_{i}(0 \leq i \leq 3)$, we have six subgroups $G_{i j}=\left\langle\sigma_{i}, \sigma_{j}\right\rangle$, where $0 \leq i<j \leq 3$. Clearly $G_{i j} \cong V_{4}$. For example, $G_{12}=\left\{\right.$ id, $\left.\sigma_{1}, \sigma_{2}, \sigma_{1} \sigma_{2}\right\}$, where $\left(\sigma_{1} \sigma_{2}\right)(z)=z+(1+\omega) / 2$.

Lemma 16. Putiing $a_{i}=\left(e_{i}-e_{j}\right)\left(e_{i}-e_{k}\right)$, we have

$$
\sigma_{0}^{*}(x)=x, \quad \sigma_{0}^{*}(y)=-y
$$


and

$$
\sigma_{i}^{*}(x)=\frac{a_{i}}{x-e_{i}}+e_{i}, \quad \sigma_{i}^{*}(y)=\frac{a_{i}}{\left(x-e_{i}\right)^{2}} y, \text { where } 1 \leq i \leq 3 .
$$

Proof. Since $x=\wp(z)$ and $y=\wp^{\prime}(z)$, we can prove them by using the the addition formulas of $\wp$ and $\wp^{\prime}$ :

$$
\begin{aligned}
\wp\left(z_{1}+z_{2}\right)= & -\wp\left(z_{1}\right)-\wp\left(z_{2}\right)+\frac{1}{4}\left(\frac{\wp^{\prime}\left(z_{1}\right)-\wp^{\prime}\left(z_{2}\right)}{\wp\left(z_{1}\right)-\wp\left(z_{2}\right)}\right)^{2} \text { and } \\
\wp^{\prime}\left(z_{1}+z_{2}\right)= & \frac{-1}{\wp\left(z_{1}\right)-\wp\left(z_{2}\right)}\left[\wp^{\prime}\left(z_{1}\right)\left\{\left(-\wp\left(z_{1}\right)-2 \wp\left(z_{2}\right)\right)+\frac{1}{4}\left(\frac{\wp^{\prime}\left(z_{1}\right)-\wp^{\prime}\left(z_{2}\right)}{\wp\left(z_{1}\right)-\wp\left(z_{2}\right)}\right)^{2}\right\}\right. \\
& +\wp^{\prime}\left(z_{2}\right)\left\{\left(2 \wp\left(z_{1}\right)+\wp\left(z_{2}\right)-\frac{1}{4}\left(\frac{\wp^{\prime}\left(z_{1}\right)-\wp^{\prime}\left(z_{2}\right)}{\wp\left(z_{1}\right)-\wp\left(z_{2}\right)}\right)^{2}\right\}\right]
\end{aligned}
$$

Since $\mathcal{L}\left(4 P_{0}\right)=\left\langle 1, x^{2}, x, y\right\rangle$, we can assume the curve $C$ is given by the embedding $\phi(x, y)=\left(1: x^{2}: x: y\right)$. Let $(X: Y: Z: W)$ be a set of homogeneous coordinates on $\mathbb{P}^{3}$. Then the ideal of $C$ is generated by

$$
F_{1}=X Y-Z^{2} \text { and } F_{2}=4 Y Z+p X Z+q X^{2}-W^{2} .
$$

Lemma 17. Using the same notation $G_{i j}=\left\langle\sigma_{i}, \sigma_{j}\right\rangle$ as in the proof of Lemma 15, we denote by $K_{i j}=k(x, y)^{G_{i j}}$ the fixed subfield of $k(x, y)$ by $G_{i j}$. Then we have

$$
K_{0 i}=k\left(\frac{x^{2}+c_{i}}{x-c_{i}}\right) \text {, where } 1 \leq i \leq 3
$$

and

$$
K_{i j}=k\left(\frac{y}{c_{k}+2 e_{k} x-x^{2}}\right), \text { where } 1 \leq i<j \leq 3 \text { and }(k-i)(k-j) \neq 0 .
$$

In particular, the Galois lines which correspond to $G_{0 i}$ and $G_{i j}$ by the arrangementmap $\rho$ are

$$
Y+c_{i} X=Z-e_{i} X=0 \text { and } c_{k} X-Y+2 e_{k} Z=W=0
$$

respectively.

Proof. By making use of Lemma 16, we can check the assertions by direct calculations.

Now we proceed with the proof of Lemma 2, Let $S=V(F)$ be a surface containing $C$. Then $F$ can be expressed as $\lambda_{1} F_{1}+\lambda_{2} F_{2}$, where $\left(\lambda_{1}: \lambda_{2}\right) \in \mathbb{P}^{1}$. In case $\lambda_{2}=0$, the point $Q_{0}=(0: 0: 0: 1)$ is the singular point of $V\left(F_{1}\right)$. On the other hand, in case $\lambda_{2} \neq 0$, put $b=\lambda_{1} / \lambda_{2}$. So we assume $F=b F_{1}+F_{2}$. Consider the condition that $V(F)$ has a singular point, i.e., consider the simultaneous linear equations

$$
F_{X}=F_{Y}=F_{Z}=F_{W}=0 .
$$

This is equivalent to consider the rank of the matrix

$$
M_{b}=\left(\begin{array}{cccc}
2 q & b & p & 0 \\
b & 0 & 4 & 0 \\
p & 4 & -2 b & 0 \\
0 & 0 & 0 & -2
\end{array}\right) .
$$


The equations (3) have a non-trivial solution if and only if

$$
b^{3}+4 p b-16 q=0 .
$$

It easy to see that the left hand side of (5) can be factored into

$\left(b+4 e_{1}\right)\left(b+4 e_{2}\right)\left(b+4 e_{3}\right)$. Thus, there exist three distinct solutions of (3). Since the rank of $M_{b}$ is three for each solution of (3), each surface $S_{i}=V\left(b_{i} F_{1}+F_{2}\right)$ is irreducible, where $b_{i}=-4 e_{i}$. Let $Q_{i}$ be the unique singular point of $S_{i}$. By simple calculations we obtain $Q_{i}=\left(8:-2 p-b_{i}{ }^{2}:-2 b_{i}: 0\right)=\left(1:-c_{i}: e_{i}: 0\right)$, where $c_{i}=e_{i}{ }^{2}+e_{j} e_{k}$ such that $\{i, j, k\}=\{1,2,3\}$. Since

$$
\operatorname{det}\left(\begin{array}{lll}
1 & -c_{1} & e_{1} \\
1 & -c_{2} & e_{2} \\
1 & -c_{3} & e_{3}
\end{array}\right)=2\left(e_{1}-e_{2}\right)\left(e_{2}-e_{3}\right)\left(e_{3}-e_{1}\right) \neq 0
$$

the four points are not coplanar. This completes the proof.

The proof of Remark 3 is as follows. Let $\Sigma_{Q}$ be the set $\left\{\ell_{Q R} \mid R \in C\right\}$. Then there exists a cone $S_{Q}$ with the singularity at $Q$ such that $S_{Q} \supset C$ and $S_{Q} \supset \Sigma$. Therefore, by Lemma 2, we have $Q=Q_{i}$ for some $i$.

Combining Lemmas 1, 2 and 15, we infer readily Theorem 1.

Remark 18. By using the condition $(\diamond)$ in Lemma 12, we can prove that the number of $V_{4}$-lines is just six. However, Lemmas 1 and 2 give the more detailed structure of the arrangement of $V_{4}$-lines.

Now we go to the proof of Theorem 2, Since $j(C)=1$, we can assume $\omega=\sqrt{-1}$. Hereafter, for simplicity we use $i$ instead of $\sqrt{-1}$, so $\mathcal{L}=\mathbb{Z}+\mathbb{Z} i$.

Lemma 19. The number of $Z_{4}$-lines is at most eight.

Proof. Suppose $C$ has a $Z_{4}$-line $\ell$. Then, let $H$ be a plane containing $\ell$ and $P_{0}{ }^{\prime}$. Since $\left.\pi_{\ell}\right|_{C}: C \longrightarrow \mathbb{P}^{1}$ is a $Z_{4}$-covering, one of the following three cases take place:

(i) $H \cdot C=4 P_{0}{ }^{\prime}$.

(ii) $H \cdot C=2 P_{0}^{\prime}+2 P_{\gamma}^{\prime}$

(iii) $H \cdot C=P_{0}{ }^{\prime}+P_{\gamma_{1}}{ }^{\prime}+P_{\gamma_{2}}{ }^{\prime}+P_{\gamma_{3}}{ }^{\prime}$.

Suppose $G=\langle\sigma\rangle$, where

$$
\sigma(z)=i z+\alpha
$$

on the universal covering $\mathbb{C}$. The case (i) occurs if and only if $P_{0}^{\prime}$ is a fixed point for $\sigma$, i.e., $\alpha \equiv 0(\bmod \mathcal{L})$ in $(6)$. The case (ii) occurs if and only if $P_{0}^{\prime}$ is a fixed point for $\sigma^{2}$, i.e., $2 \alpha \equiv 0(\bmod \mathcal{L})$ in (6) $)$. Concerning the last case (iii), since $H \cdot C$ is invariant by the action of $G$, it can be expressed as $P_{0}{ }^{\prime}+P_{\alpha}{ }^{\prime}+P_{i \alpha}{ }^{\prime}+P_{(1+i) \alpha}{ }^{\prime}$. Since this is linearly equivalent to $4 P_{0}{ }^{\prime}$, we infer

$$
P_{\alpha}+P_{i \alpha}+P_{(1+i) \alpha} \sim 3 P_{0}
$$

on the curve $E$. Moreover the left hand side of (7) is linearly equivalent to $P_{2(1+i) \alpha}+$ $2 P_{0}$ by Lemma 8 . Therefore we have $P_{2(1+i)} \alpha \sim P_{0}$. This implies $2(1+i) \alpha \equiv 0$ $(\bmod \mathcal{L})$. To find the possibility of $\alpha$, it is sufficient to solve the equation $2(1+i) \alpha \equiv$ $0(\bmod \mathcal{L})$. By a simple calculation we have $\alpha=(m+n i) / 4$, where

$$
(m, n)=(0,0),(2,2),(2,0),(0,2),(3,1),(1,3),(1,1),(3,3) .
$$


Thus we get eight subgroups, which might be the images of $\rho$ of Definition 3 .

Checking the condition $(\diamond)$ of Lemma 12, we now prove Theorem 2, As we see from the proof of Lemma 19, we have $G=\langle\sigma\rangle$, where $\sigma(z)=i z+\alpha$. Since $\sigma$ has fixed points, the curve $C / G$ is rational. For each point $Q \in C$ there exists $\gamma \in \mathbb{C}$ satisfying that $Q=P_{\gamma}^{\prime}$. So it is sufficient to prove that $P_{\gamma}{ }^{\prime}+P_{\sigma(\gamma)}{ }^{\prime}+P_{\sigma^{2}(\gamma)}{ }^{\prime}+P_{\sigma^{3}(\gamma)^{\prime}} \sim 4 P_{0}{ }^{\prime}$. Since $2(1+i) \alpha \equiv 0(\bmod \mathcal{L})$ as in the proof of Lemma 19 , this holds true by Lemma 8. Since $j(C)=1$, we can assume $y^{2}=4 x^{3}-x$ and hence $e_{1}=1 / 2, e_{2}=$ $-1 / 2, e_{3}=0$. Thus we have $Q_{0}=(0: 0: 0: 1), Q_{1}=(4:-1: 2: 0), Q_{2}=(4:$ $-1:-2: 0)$ and $Q_{3}=(4: 1: 0: 0)$. Let $\ell_{1}$ and $\ell_{2}$ are $Z_{4}$-lines and $G_{\ell_{1}}=\left\langle\tau_{1}\right\rangle$ and $G_{\ell_{2}}=\left\langle\tau_{2}\right\rangle$. If $\ell_{1}$ and $\ell_{2}$ meet, then we have $\tau_{1}{ }^{2}=\tau_{2}{ }^{2}$ by Lemma 13. Letting $\tau_{1}(z)=i z+\alpha_{1}$ and $\tau_{2}(z)=i z+\alpha_{2}$, we have $(1+i)\left(\alpha_{1}-\alpha_{2}\right) \in \mathcal{L}$. Denote by $\ell(m, n)$ the line corresponding to the group $\langle\tau\rangle$ by the arrangement-map $\rho$, where $\tau(z)=i z+(m+n i) / 4$. The following assertion is easy to see.

Claim 1. Putting $\sigma_{m n}(z)=i z+(m+n i) / 4$ and $G_{m n}=\left\langle\sigma_{m n}\right\rangle$, we have $G_{00} \cap G_{22}=$ $\left\langle\sigma_{0}\right\rangle, G_{20} \cap G_{02}=\left\langle\sigma_{3}\right\rangle, G_{11} \cap G_{33}=\left\langle\sigma_{2}\right\rangle$ and $G_{31} \cap G_{13}=\left\langle\sigma_{1}\right\rangle$.

Claim 2. The intersections of the eight $Z_{4}$-lines are $\ell(0,0) \cap \ell(2,2)=Q_{0}, \ell(2,0) \cap$ $\ell(0,2)=Q_{3}, \ell(1,1) \cap \ell(3,3)=Q_{2}$ and $\ell(3,1) \cap \ell(1,3)=Q_{1}$.

Proof. The intersection points are found by Lemma 17. For example, the point $\ell(1,1) \cap \ell(3,3)$ is found as follows: Since $G_{11} \cap G_{33}=\left\langle\sigma_{2}\right\rangle$, the point is the intersection of two lines

$$
c_{3} X-Y+2 e_{1} Z=W=0 \text { and } c_{1} X-Y+2 e_{1}=W=0,
$$

where $e_{1}=1 / 2, e_{3}=0$ and $c_{1}=1 / 4, c_{3}=-1 / 4$. So it is $Q_{2}$.

Now, we prove Corollary 5 . Let $E$ be the Weierstrass normal form of the normalization of $\Gamma$ and let $\mu: E \longrightarrow \Gamma \subset \mathbb{P}^{3}$ be the normalization morphism. Put $D=\mu^{*}(L)$ for a line $L$ in $\mathbb{P}^{2}$. By Lemma 10 we can assume $C$ is given by the embedding by $\left|4 P_{0}\right|$. Therefore, $\Gamma$ is regained as $\pi_{P}(C)$, where $\pi_{P}: \mathbb{P}^{3} \rightarrow \mathbb{P}^{2}$ is the projection with center $P$. Suppose $\Gamma$ has two Galois points $Q_{1}$ and $Q_{2}$. Then, letting $\ell_{1}=\pi_{P}{ }^{*}\left(Q_{1}\right)$ and $\ell_{2}=\pi_{P}{ }^{*}\left(Q_{2}\right)$, they are Galois lines for $C$ and $\ell_{1} \cap \ell_{2}=\{P\}$. However, as we have seen Remark 14, the projection $\pi_{P}$ induces a 2 to 1 morphism from $C$ to $\Gamma$ and $\pi_{P}(C)$ is a rational curve, this is a contradiction. On the other hand, if $P$ lies in one of the Galois lines, i.e., $P \in \ell$ and is not the vertex, then $\pi_{P}$ induces a birational transformation on $C$ by Remark 3 and $\pi_{P}(\ell \backslash\{P\})$ is a Galois point for $\Gamma=\pi_{P}(C)$.

Finally, we mention Remark 6. Take a point $Q \in \mathbb{P}^{3}$ which does not lie on the Galois lines. Then, the curve $\Gamma=\pi_{Q}(C)$ is a quartic curve with no Galois point. Because, by Remark 3 it is birational to $C$. Suppose it has a Galois point. Then, there exists a smooth quartic curve $C^{\prime}$ in $\mathbb{P}^{3}$ and a Galois line $\ell^{\prime}$ and a point $P^{\prime} \in \mathbb{P}^{3}$ satisfying that $\pi_{P^{\prime}}\left(C^{\prime}\right)=\Gamma$. Moreover, there exists an isomorphism $\varphi: \mathbb{P}^{3} \longrightarrow \mathbb{P}^{3}$ such that $\varphi\left(C^{\prime}\right)=C$ and $\varphi\left(\ell^{\prime}\right)$ coincides with some Galois line for $C$. Since $\ell^{\prime} \ni P^{\prime}$, we have $\varphi\left(\ell^{\prime}\right) \ni P$, which is a contradiction.

Thus we complete all proofs. 
Problem. We ask the following questions concerning Galois embedding of elliptic curves.

(a) In case $\ell$ is not a Galois line, consider the Galois group $G$ of the Galois closure curve [5, Definition 1.3]. If $\ell$ is general, then the Galois group is a full symmetric group [5, Theorem 2.2], see also [4]. So we ask if $\ell$ is neither general (i.e., $G \neq S_{4}$ ) nor Galois, then what group can appear. For the group which appears, how are the arrangements of the lines with the group?

(b) Let $D$ be a divisor of degree $d \geq 5$ on $E$. Then, study the Galois embedding by $|D|$. In particular, consider the Galois group and the arrangement of Galois subspaces ([6]).

\section{REFERENCES}

1. C. Duyaguit and H. Yoshihara, Galois lines for normal elliptic space curves, Algebra Colloquium, 12 (2005), 205-212.

2. R. Hartshorne, Algebraic Geometry, Graduate Texts in Math., 52 (1977), Springer-Verlag.

3. K. Miura, Galois points on singular plane quartic curves, J. Algebra, 287 (2005), 283-293.

4. G. P. Pirola and E. Schlesinger, Monodromy of projective curves, J. Algebraic Geometry, 14 (2005), 623-642.

5. H. Yoshihara, Galois lines for space curves, Algebra Colloquium, 13 (2006), 455-469.

6. Galois embedding of algebraic variety and its application to abelian surface, Rend. Sem. Mat. Universita di Padova 117 (2007), 69-85. 